\title{
A centrifugation-based clearing method allows high-throughput acidification and growth-rate measurements in milk
}

\author{
Sieze Douwenga, ${ }^{1,2}$ Patrick Janssen, ${ }^{1,3}$ Bas Teusink, ${ }^{1,2} \odot$ and Herwig Bachmann ${ }^{1,2,3 *}$ (]) \\ ${ }^{1} \mathrm{TI}$ Food and Nutrition, 6709 PA, Wageningen, the Netherlands \\ ${ }^{2}$ Systems Biology Lab, Vrije Universiteit Amsterdam, $1081 \mathrm{HZ}$, Amsterdam, the Netherlands \\ ${ }^{3}$ Health Department, NIZO Food Research, 6718 ZB, Ede, the Netherlands
}

\begin{abstract}
The turbidity of milk prohibits the use of optical density measurements for strain characterizations. This often limits research to laboratory media. Here, we cleared milk through centrifugation to remove insoluble milk solids. This resulted in a clear liquid phase, termed milk serum, in which optical density measurements can be used to track microbial growth until a $\mathrm{pH}$ of 5.2 is reached. At pH 5.2 coagulation of the soluble protein occurs, making the medium opaque again. We found that behavior in milk serum was predictive of that in milk for 39 Lactococcus lactis $\left(\mathrm{R}^{2}=0.81\right)$ and to a lesser extent for 42 Lactiplantibacillus plantarum (formerly Lactobacillus plantarum; $\mathrm{R}^{2}=0.49$ ) strains. Hence, milk serum can be used as an optically clear alternative to milk for comparison of microbial growth and metabolic characteristics. Characterization of the growth rate, specific acidification rate for optical density at a wavelength of $600 \mathrm{~nm}$, and the amount of acid produced per unit of biomass for all these strains in milk serum, showed that almost all strains could grow in milk, with higher specific acidification and growth rates of Lc. lactis strains compared with $L b$. plantarum strains. Nondairy Lc. lactis isolates had a lower growth and specific acidification rate than dairy isolates. The amount of acid produced per unit biomass was relatively high and similar for Lc. lactis dairy and nondairy isolates, as opposed to $L b$. plantarum isolates. Lactococcus lactis ssp. lactis showed slightly lower growth and acidification rates when compared with ssp. cremoris. For Lc. lactis strains a doubling of the specific acidification rate occurred with a doubling of the maximum growth rate. This relation was not found for $L b$. plantarum strains, where the acidification rate remained relatively constant across 39 strains with growth rates ranging from $0.2 \mathrm{~h}^{-1}$ to $0.3 \mathrm{~h}^{-1}$. We conclude that milk serum
\end{abstract}

Received December 29, 2020.

Accepted March 25, 2021.

*Corresponding author: Herwig.Bachmann@nizo.com is a valuable alternative to milk for high-throughput strain characterization during milk fermentation.

Key words: milk serum, milk clearing, growth rate, optical density, acidification rate

\section{INTRODUCTION}

Microbes play a key role in the dairy industry, with $37.7 \%$ of the produced milk ( 172.2 billion $\mathrm{kg}$ ) being fermented to cheese in the European Union alone in 2018 (Silva et al., 2019). The starter cultures used during fermentation are often composed of numerous strains; they are responsible for the conversion of lactose into lactic acid and production of flavor compounds, and they affect the texture of the final product. There are big genotypic and phenotypic differences between microorganisms in starter cultures (Siezen et al., 2011; Wels et al., 2019), which causes differences in specific acidification rates, growth rates, and lag phases during industrial fermentations of milk. Quantifying these traits for different strains in milk can be challenging as the turbidity of milk does not allow for online monitoring of cell growth, which is typically done through optical density (OD) measurements.

Multiple solutions to determine the cell concentration in milk exist, one of which is the standard plate count. However, this process is laborious and typically takes more than a day, and counts can be imprecise. Fowler et al. (1978) report 18\% cell count variation between analysts, and $7 \%$ variation for repetitions by one analysist. However, precision can improve with experience and training of the analyst (Fruin et al., 1977). Similar findings were also reported in (Jarvis et al., 2007). A faster $(\leq 1 \mathrm{~h})$ method was developed by (Gunasekera et al., 2000). Casein micelles are enzymatically digested, allowing them to dissolve. Cells are then separated from the milk by centrifugation. This allows cell staining and subsequent cell counting using flow cytometry. The digestive enzyme must be carefully selected, however, as not all cells remain culturable when savinase is used (Smith et al., 2003; Meisel et al., 2011). In an even faster protocol $(\sim 5 \mathrm{~min})$, the milk is cleared by 
mixing it with a solution of $0.2 \%$ sodium hydroxide and $0.2 \%$ EDTA, dissolving the suspended casein micelles. This allows OD measurements as an indirect measure for cell density (Bachmann et al., 2012). Meisel et al. (2011) separate cells from milk using buoyant density centrifugation. Their method is relatively quick $(\leq 1 \mathrm{~h})$, and does not require chemicals, but it recovers only $35 \%$ of microorganisms in the milk. Hence, although cell densities can be determined in milk, current methods can be labor intensive, inaccurate, give the readout with long delays, are destructive to the sample, and are therefore unsuited for continuous online monitoring.

Although continuous growth monitoring of the cell count is currently difficult, acidification of the environment can be measured continuously and is used to detect metabolic activity of lactic acid bacteria. Acidification can be monitored either directly with $\mathrm{pH}$ probes or indirectly through the use of fluorescent $\mathrm{pH}$ sensitive dyes. The dyes allow acidification tracking of many cultures in parallel using multiwell plates (John et al., 2003; Wegkamp and Reemst, 2018; Nugroho et al., 2020). Acidification is a function of the cell density and biomass specific acidification rate. Therefore, acidification is only a proxy for growth and cannot be used to determine the exact growth rate, lag phase, lactic acid production per unit biomass, or specific acidification rate of the culture being analyzed.

We developed a protocol to clear milk and use the obtained serum for the simultaneous high-throughput online analysis of both the growth rate through OD and the $\mathrm{pH}$ through the use of a fluorescent probe. This facilitated accurate analysis of growth rate, lag phase, and specific acidification rates in milk. The protocol was validated for 39 Lactococcus lactis and 42 Lactiplantibacillus plantarum strains (the latter being formerly known as Lactobacillus plantarum; Zheng et al., 2020), showing that acidification profiles in milk and milk serum were similar.

\section{MATERIALS AND METHODS}

\section{Strains and Media}

The strains used in this study are listed in Table 1 . All strains were taken from the NIZO culture collection (NIZO). The majority of Lc. lactis strains are based on a set described by Rademaker et al. (2007) and Brooijmans (2008). The Lb. plantarum strains are described in (Molenaar et al., 2005; Smits et al., 2005; Siezen et al., 2010). Skimmed milk (Tritium Microbiologie) from the same batch was used for all growth experiments. This milk was reconstituted from powdered skim milk and autoclaved at $115^{\circ} \mathrm{C}$ for $10 \mathrm{~min}$. The term milk throughout the paper refers to skim milk prepared this way.

Lactococcus lactis and Lb. plantarum strains were precultured in a 96-well plate (Greiner Bio-One) at $30^{\circ} \mathrm{C}$ and $37^{\circ} \mathrm{C}$ for $16 \mathrm{~h}$, in $0.5 \%$ glucose M17 (Tritium Microbiologie; Oxoid) and MRS broth (Tritium Microbiologie; Merck), respectively. For acidification measurements, milk and milk serum were inoculated with $0.2 \% \mathrm{vol} / \mathrm{vol}$ of the stationary preculture.

\section{Milk Clearing and Acidification Measurements}

Milk serum was prepared by centrifuging a total of $650 \mathrm{~mL}$ milk divided over 16 sterile metal centrifuge tubes, with $40 \mathrm{~mL}$ of milk in each tube. A Beckman Coulter Avanti J26XP centrifuge was run with a Beckman Coulter JA-14.50 rotor at $35,000 \times g$ at $10^{\circ} \mathrm{C}$ for 1 $\mathrm{h}$, maximum acceleration, and slow deceleration. This results in solid precipitate below and a thin layer of solids on top of the supernatant. The top $30 \mathrm{~mL}$ of the supernatant was harvested immediately upon stopping of the centrifuge. Care was taken to transfer as little as possible of the top layer of solids by sticking the pipet through this surface before aspiration, although this has little effect on the turbidity of the milk serum. The harvested supernatant was then again divided over sterile centrifuge tubes, $40 \mathrm{~mL} /$ tube, and centrifuged with the same settings. This time $25 \mathrm{~mL}$ supernatant was removed and pooled in a sterile bottle. The pooled milk serum (supernatant) was stored at $4^{\circ} \mathrm{C}$ until further use. For all experiments milk serum from the same batch was used.

To be able to measure $\mathrm{pH}$ through fluorescence, both milk serum and milk were supplemented with 1:1,000 of $0.4 \% \mathrm{wt} / \mathrm{vol} 5(6)$-Carboxyfluorescein BioReagent (Sigma-Aldrich) suspended in $10 \% \mathrm{vol} / \mathrm{vol}$ ethanol in demineralized water [final 5(6)-carboxyfluorescein concentration of $0.0004 \% \mathrm{wt} / \mathrm{vol}$; Bachmann et al., 2013]. Carboxyfluorescein fluorescence positively correlates with the $\mathrm{pH}$. Milk and milk serum from the same batch were used for all experiments.

After preculture, strains were diluted $20 \times$ in phosphate buffer (100 $\mathrm{m} M, \mathrm{pH}$ 6.5) and subsequently another $26 \times$ in milk or milk serum $(520 \times$ final dilution), in 2 new 96-well (300 $\mu \mathrm{L} /$ well) plates (Greiner Bio-One). The inoculated milk and milk serum were transferred to a black 384 -well plate $(100 \mu \mathrm{L} /$ well $)$ with a transparent bottom (Greiner Bio-One). The plate was placed in a plate reader (Tecan Safire II) at $30^{\circ} \mathrm{C}$ without shaking, where fluorescence (excitation: 485/20 $\mathrm{nm}$; emission: $520 / 20 \mathrm{~nm}$ ) and OD at a wavelength of $600 \mathrm{~nm}\left(\mathbf{O D}_{600}\right)$ were measured every $6 \mathrm{~min}$ for $42 \mathrm{~h}$. The milk and milk serum $\mathrm{pH}$ and proton production 
Table 1. Lactococcus and Lactiplantibacillus strains used throughout this study, including their phenotypic characterization and origin $^{1}$

\begin{tabular}{|c|c|c|c|}
\hline Genotype & Phenotype & Strain & Origin \\
\hline Lactococcus lactis ssp. cremoris & lactis & NCDO763 & Dairy \\
\hline Lc. lactis ssp. lactis & lactis & $\mathrm{R} 1$ & Dairy \\
\hline Lc. lactis ssp. lactis & lactis & ML8 & Dairy \\
\hline Lc. lactis ssp. lactis & lactis & ATCC19435 & Dairy \\
\hline Lc. lactis ssp. cremoris & cremoris & SK11 & Dairy \\
\hline Lc. lactis ssp. cremoris & lactis & AM2 & Dairy \\
\hline Lc. lactis ssp. lactis & lactis & LMG8526 & Nondairy \\
\hline Lc. lactis ssp. lactis biovar diacetylactis & & M20 & Nondairy \\
\hline Lc. lactis & Unknown & M9 & Nondairy \\
\hline Lc. lactis ssp. lactis & lactis & E7 & Nondairy \\
\hline Lc. lactis ssp. lactis & lactis & $\mathrm{Li}-1$ & Nondairy \\
\hline Lc. lactis $\mathrm{ssp}$. cremoris & cremoris & $\mathrm{P} 2$ & Dairy \\
\hline Lc. lactis ssp. cremoris & cremoris & $\mathrm{HP}$ & Dairy \\
\hline Lc. lactis ssp. cremoris & cremoris & AM1 & Dairy \\
\hline Lc. lactis ssp. cremoris & cremoris & $\mathrm{KH}$ & Dairy \\
\hline Lc. lactis ssp. cremoris & cremoris & E8 & Dairy \\
\hline Lc. lactis ssp. cremoris & cremoris & Wg2 & Dairy \\
\hline Lc. lactis ssp. lactis & lactis & E30 & Nondairy \\
\hline Lc. lactis ssp. lactis & lactis & E33 & Nondairy \\
\hline Lc. lactis ssp. lactis & lactis & E34 & Nondairy \\
\hline Lc. lactis ssp. lactis & lactis & E32 & Nondairy \\
\hline Lc. lactis ssp. cremoris & lactis & N41 & Nondairy \\
\hline Lc. lactis ssp. lactis & lactis & N42 & Nondairy \\
\hline Lc. lactis ssp. lactis biovar diacetylactis & & $\mathrm{ZK}$ & Dairy \\
\hline Lc. lactis ssp. lactis & lactis & UC317 & Dairy \\
\hline Lc. lactis ssp. cremoris & lactis & MG1363 & Dairy \\
\hline Lc. lactis ssp. lactis & lactis & E10 & Nondairy \\
\hline Lc. lactis & Unknown & $\mathrm{E} 27$ & Nondairy \\
\hline Lc. lactis ssp. cremoris & cremoris & E9 & Nondairy \\
\hline Lc. lactis ssp. lactis & lactis & LMG8528 & Nondairy \\
\hline Lc. lactis ssp. lactis & lactis & LMG9446 & Nondairy \\
\hline Lc. lactis ssp. lactis biovar diacetylactis & & C17 & Dairy \\
\hline Lc. lactis ssp. lactis biovar diacetylactis & & B35 & Dairy \\
\hline Lc. lactis ssp. lactis & lactis & D53 & Nondairy \\
\hline Lc. lactis ssp. lactis & lactis & $\mathrm{K} 231$ & Nondairy \\
\hline Lc. lactis ssp. lactis & lactis & K335 & Nondairy \\
\hline Lc. lactis ssp. lactis & lactis & K336 & Nondairy \\
\hline Lc. lactis ssp. lactis & lactis & K337 & Nondairy \\
\hline Lc. lactis ssp. lactis & lactis & K338 & Nondairy \\
\hline Lactiplantibacillus plantarum & & WCFS1 & Nondairy \\
\hline Lb. plantarum & & ATCC 14917 & Nondairy \\
\hline Lb. plantarum & & MLC43 & Dairy \\
\hline Lb. plantarum & & CHEO3 & Nondairy \\
\hline Lb. plantarum & & NCTH19-1 & Nondairy \\
\hline Lb. plantarum & & NCTH19-2 & Nondairy \\
\hline Lb. plantarum & & NCTH27 & Nondairy \\
\hline Lb. plantarum & & LD2 & Nondairy \\
\hline Lb. plantarum & & NOS140 & Nondairy \\
\hline Lb. plantarum & & Q2 & Nondairy \\
\hline Lb. plantarum & & $\mathrm{H} 4$ & Nondairy \\
\hline Lb. plantarum & & H14 & Nondairy \\
\hline Lb. plantarum & & L9 & Dairy \\
\hline Lb. plantarum & & KOG18 & Nondairy \\
\hline Lb. plantarum & & KOG24 & Dairy \\
\hline Lb. plantarum & & LMG9208 & Nondairy \\
\hline Lb. plantarum & & Lp95 & Nondairy \\
\hline Lb. plantarum & & BLL & Nondairy \\
\hline Lb. plantarum & & CECT221 & Nondairy \\
\hline Lb. plantarum & & N58 & Nondairy \\
\hline Lb. plantarum & & X17 & Nondairy \\
\hline Lb. plantarum & & $\mathrm{LAC} 7$ & Nondairy \\
\hline Lb. plantarum & & LD3 & Nondairy \\
\hline Lb. plantarum & & $\mathrm{DKO} 22$ & Nondairy \\
\hline Lb. plantarum & & 299 & Nondairy \\
\hline Lb. plantarum & & CIP104440 & Nondairy \\
\hline
\end{tabular}


Table 1 (Continued). Lactococcus and Lactiplantibacillus strains used throughout this study, including their phenotypic characterization and origin ${ }^{1}$

\begin{tabular}{|c|c|c|c|}
\hline Genotype & Phenotype & Strain & Origin \\
\hline Lb. plantarum & & SF2A35B & Nondairy \\
\hline Lb. plantarum & & NCIMB12120 & Nondairy \\
\hline Lb. plantarum & & CIP104441 & Nondairy \\
\hline Lb. plantarum & & CIP1044550 & Nondairy \\
\hline Lb. plantarum & & CIP104451 & Nondairy \\
\hline Lb. plantarum & & CIP104452 & Nondairy \\
\hline Lb. plantarum & & $299 \mathrm{v}$ & Nondairy \\
\hline Lb. plantarum & & NC8 & Nondairy \\
\hline Lb. plantarum & & LM3 & Nondairy \\
\hline Lb. plantarum & & LP80 & Nondairy \\
\hline Lb. plantarum & & LP85-2 & Nondairy \\
\hline Lb. plantarum & & ATCC8014 & Nondairy \\
\hline Lb. plantarum & & NCDO1193 & Nondairy \\
\hline Lb. plantarum & & CIP102359 & Nondairy \\
\hline Lb. plantarum & & CIP104448 & Nondairy \\
\hline Lb. plantarum & & Unknown & Dairy \\
\hline
\end{tabular}

${ }^{1}$ All strains were obtained from the NIZO culture collection.

were determined from fluorescence measurements in combination with calibration curves.

\section{Calibration Curves}

We determined the relation between fluorescence, $\mathrm{pH}$, proton production, and turbidity (as a cause of increasing casein precipitation at decreasing $\mathrm{pH})$. To this end milk and milk serum [both with 5(6)-carboxyfluorescein] at varying concentrations of lactic acid were prepared by titrating known amounts of $0.484 \mathrm{M}$ lactic acid into $10 \mathrm{~mL}$ of milk and milk serum. The $\mathrm{pH}$ was determined using a $\mathrm{pH}$ probe (Unitrode, Metrohm AG). The different solutions were added to a black 384well plate and fluorescence and $\mathrm{OD}_{600}$ were measured in a plate reader.

\section{Data Analysis}

Data analysis was done using Python v3.7.4. Packages used were: pandas v1.0.3 (McKinney, 2010), numpy v1.18.1 (van der Walt et al., 2011), matplotlib v3.1.2 (Hunter, 2007), seaborn v0.10.1 (Waskom et al., 2020), and scipy v1.4.1 (Virtanen et al., 2020).

The relation of fluorescence to $\mathrm{pH}$ and proton production was determined by fitting least squares second order polynomials to the calibration curves for milk and milk serum in each plate (adapted from Nugroho et al., 2020). Numpy's polyfit algorithm was used.

In all cases where $\mathrm{OD}_{600}$ was measured in milk serum, before further data analysis the background $\mathrm{OD}_{600}$ was subtracted. The background was determined in wells containing sterile milk serum.

Continuous growth rates $[\mu(\mathrm{t})]$ were determined using a savitsky-golay filter (Savitzky and Golay, 1964) on the natural logarithm of the $\mathrm{OD}_{600}$ data versus time. The filter was set to fit a first order polynomial over a measurement window of $2 \mathrm{~h}$, returning the first derivative as the growth rate. The maximal growth rate $\left(\boldsymbol{\mu}_{\max }\right)$ was determined from the continuous growth rates at $\mathrm{OD}_{600}>0.024$ and at lactic acid concentrations $<9.2 \mathrm{~m} M$ (to rule out the effect background noise and casein precipitation, respectively). Additionally, at these lactic acid concentrations the $\mathrm{pH}$ was higher than 5.5, meaning almost all lactic acid dissociates $\left[\mathrm{p} K_{\mathrm{a}}\right.$ $($ acid dissociation constant $)=3.8]$, making lactic acid addition equal to $\mathrm{H}^{+}$addition.

Acid production per unit produced $\mathrm{OD}_{600}\left(\mathrm{Y}_{\mathrm{H} / \mathrm{OD}}\right)$ was determined by fitting least squared first order polynomials to $\mathrm{H}^{+}$versus $\mathrm{OD}_{600}$ curves where their relation is linear (at: $0.02<\mathrm{OD}_{600}<0.08$ ). Numpy's polyfit algorithm was used. Maximum specific acidification rates $\left(\mathrm{qH}^{+}\right)$were determined by multiplying $\mu_{\max }$ with $\mathrm{Y}_{\mathrm{H} / \mathrm{OD}}$. When relevant the squared Pearson correlation coefficient $\left(\mathrm{R}^{2}\right)$ and corresponding 2 -tailed $P$-value were calculated with the scipy stats pearsonr function, which assumes normally distributed data.

\section{RESULTS}

\section{Removing the Insoluble Fraction of Milk Drastically Reduces the Background $O D_{600}$}

Milk is too turbid to measure cell growth using $\mathrm{OD}_{600}$. We centrifuged the milk at $35,000 \times g$ at $10^{\circ} \mathrm{C}$ for $1 \mathrm{~h}$, took the supernatant and centrifuged this again at the same settings to separate the colloidal milk particles from the serum. This reduced the $\mathrm{OD}_{600}$ from $>4$ (outside the detection range) to 0.38 . This $\mathrm{OD}_{600}$ is low enough to allow tracking of microbial growth 
through periodic $\mathrm{OD}_{600}$ measurements. In milk serum, $\mathrm{OD}_{600}$ measurements increased linearly with increasing cell density up to an $\mathrm{OD}_{600}$ of 0.66 . The pelleted particles are likely to contain a lot of micellar casein, and perhaps some other nutrients.

\section{Removal of the Insoluble Fraction of Milk Slightly Reduces the Growth Rate}

We wondered if the removal of the colloidal particles affected growth of microorganisms in milk. In addition to $\mathrm{OD}_{600}$ measurements, which are only useful for milk serum, we also indirectly monitored $\mathrm{pH}$ over time. This was done by adding a $\mathrm{pH}$-sensitive fluorescent probe to milk and milk serum. Calibration curves (Supplemental Figure S1, https://doi.org/10.5281/zenodo.4694160; Douwenga et al., 2021) allowed the conversion of the fluorescence signal to $\mathrm{pH}$ profiles and the total amount of protons produced. Normally, the slope of a logarithmic $\mathrm{OD}_{600}$ curve can be used to determine the growth rate. In milk, this is not possible, but the $\mathrm{H}^{+}$concentration should also increase logarithmically as long as the $\mathrm{H}^{+}$production per $\mathrm{OD}_{600}$ increase is constant in time. Hence, if the insoluble fraction of milk does not contribute to growth, the slopes of logarithmic $\mathrm{H}^{+}$curves in milk and milk serum of each strain are expected to be equal.

Using the methods described above, we determined the difference between acidification in milk and milk serum for $40 \mathrm{Lc}$. lactis and $42 \mathrm{Lb}$. plantarum strains. The lactococcal strains used include dairy and nondairy isolates of subspecies cremoris and subspecies lactis, to validate the method for a broad range of strains. A comparison of the measurements in milk and milk serum for Lc. lactis NCDO763 shows that the $\mathrm{pH}$ profiles are very similar (Figure 1A). pH profiles of other strains show a comparable similarity between milk and milk serum, although in some cases the final $\mathrm{pH}$ in milk serum is slightly lower $(<0.3 \mathrm{pH}$ points) (Supplemental Figure S2, https://doi.org/10.5281/zenodo.4694160; Douwenga et al., 2021). For Lb. plantarum strains, the final $\mathrm{pH}$ in milk serum is always slightly lower than that in milk (roughly $0.3 \mathrm{pH}$ points; Supplemental Figure S3, https://doi.org/10.5281/zenodo.4694160; Douwenga et al., 2021). As carboxyfluorescein becomes less sensitive to $\mathrm{pH}$ changes below $\mathrm{pH} 5$, further $\mathrm{pH}$ decreases are difficult to determine (Supplemental Figure S4, https://doi.org/10.5281/zenodo.4694160; Douwenga et al., 2021). The $\mathrm{H}^{+}$production profiles show some differences (Figure 1B), but considering that growth is exponential, these differences can be the result from only minor growth-rate differences. The slopes of the $\log \mathrm{H}^{+}$production rates (Figure 1C) differ by around $24 \%\left(0.96 \mathrm{~h}^{-1}\right.$ in milk vs. $0.76 \mathrm{~h}^{-1}$ in milk serum). The
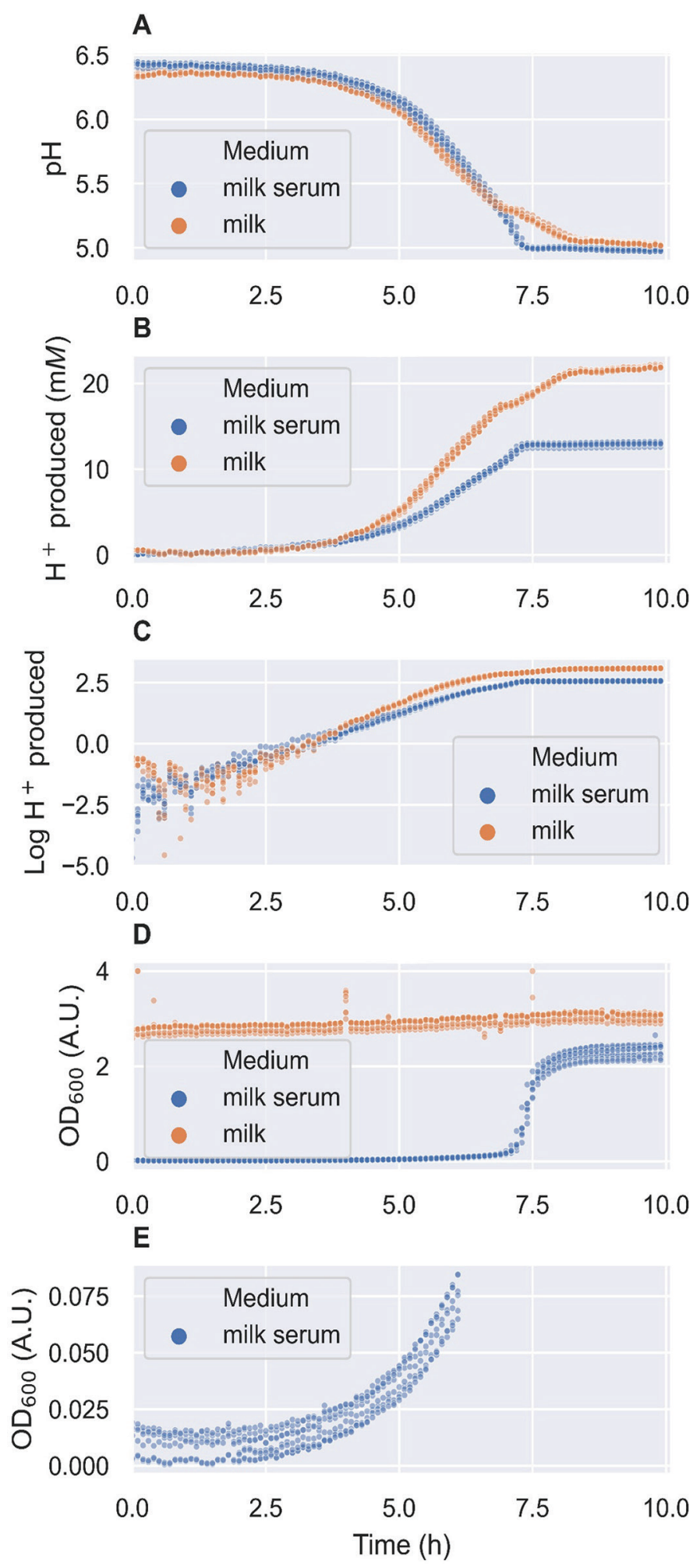

Figure 1. $\mathrm{pH}(\mathrm{A}), \mathrm{H}^{+}$produced (B), $\log \mathrm{H}^{+}(\mathrm{C})$, optical density at a wavelength of $600 \mathrm{~nm}\left(\mathrm{OD}_{600}\right)(\mathrm{D})$, and $\mathrm{OD}_{600}$ at $\mathrm{pH}>5.4(\mathrm{E}$, same data as $\mathrm{D}$, but zoomed in on the y-axis 50 times) versus time for Lactococcus lactis NCDO763 grown in milk (orange, $\mathrm{n}=4$ ) and milk serum (blue, $\mathrm{n}=4$ ). A.U. = absorbance units. Measurement frequency $=10 \mathrm{~h}^{-1}$. 
higher cumulative proton production with similar $\mathrm{pH}$ profiles indicates that milk has a higher buffer capacity than milk serum. This is consistent with the calibration curve $\mathrm{pH}$ versus lactic acid (Supplemental Figure S5, https://doi.org/10.5281/zenodo.4694160; Douwenga et al., 2021).

Growth rates were determined from $\mathrm{OD}_{600}$ measurements in the milk serum. For this, only $\mathrm{OD}_{600}$ values were used where the amount of $\mathrm{H}^{+}$produced was less than $9 \mathrm{~m} M$ (Figure 1D, E). At higher $\mathrm{H}^{+}$levels, casein starts precipitating, resulting in a sharp $\mathrm{OD}_{600}$ increase (observed after approximately $7 \mathrm{~h}$ for Lc. lactis NCDO763; Figure 1D and Supplemental Figure S6, https://doi.org/10.5281/zenodo.4694160; Douwenga et al., 2021).

Based on $\log \mathrm{H}^{+}$production rates, there appears to be an effect on growth when the insoluble fraction of milk is removed; behavior in milk serum is predictive of behavior in milk for Lc. lactis $\left(\mathrm{R}^{2}=0.81, P<0.001\right)$ and to a lesser extent for Lactiplantibacillus plantarum $\left(\mathrm{R}^{2}=0.49, P<0.001\right)$ strains (see Figure 2 and Supplemental Figures S7 and S8, https://doi.org/10.5281/ zenodo.4694160; Douwenga et al., 2021).

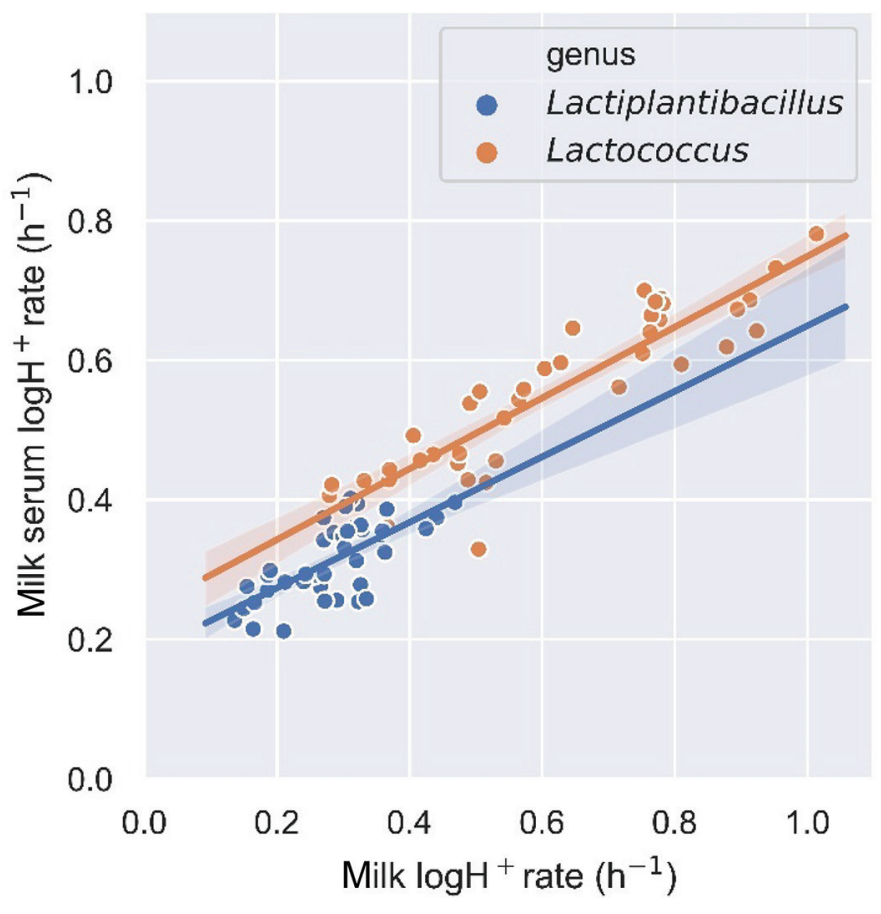

Figure 2. Relations between $\log \mathrm{H}^{+}$rates, i.e., the slopes of a $\log \mathrm{H}^{+}$ curve, in milk (x-axis) and milk serum (y-axis), for Lactiplantibacillus (blue) and Lactococcus (orange). Lactiplantibacillus $\mathrm{R}^{2}=0.49$; Lactococcus $\mathrm{R}^{2}=0.81$. Each point represents a strain average of 4 replicates (in some cases $2-3$ replicates when $R^{2}<0.9$ of the linear fit to the $\log \mathrm{H}^{+}$data).

\section{Strain Characterization Through $O D_{600}$ and $H^{+}$Measurements}

The OD measurements act as a rough estimate of cell density, as the relation between these measurements and cell density can vary between strains and growth environment. However, OD measurements are useful for rapid screening of large numbers of strains or growth environments. Slower, more accurate methods can be used to further characterize the strains of interest. To illustrate the rapid screening capacity of milk serum, we determined the biological parameters: $\mu_{\max }, \mathrm{OD}_{600^{-}}$ specific acidification rate $\left(\mathrm{qH}^{+}\right)$, and acid produced per unit $\mathrm{OD}_{600}$ (i.e., the acid on biomass yield). We studied these parameters for different lactic acid bacteria and different strain origins (dairy and nondairy). All the $L b$. plantarum strains could grow in milk with low specific acidification rates, regardless of their origin (Figure 3A, B). For Lc. lactis ssp lactis, their origin did have a large influence in most cases. For most of the nondiary isolates, the growth rate and acidification rate were lower than that of the average dairy isolate (Figure 3A, B). The amount of acid produced per unit biomass, however, remained relatively high for Lc. lactis nondairy isolates. This indicates that a major metabolic by-product is still acidic, in contrast to $L b$. plantarum strains, which produce less acid per unit biomass (Figure $3 \mathrm{C}$ ). The specific acidification rate increases with the growth rate only for Lc. lactis.

The specific acidification and maximal growth rates allowed us to determine whether these 2 parameters correlated for Lc. lactis and Lb. plantarum strains. Figure $4 \mathrm{~A}$ shows this relation for all strains tested. For Lc. lactis, the specific acidification rate doubles with a doubling in the growth rate, $\mathrm{R}^{2}=0.89, P=4 \mathrm{E}^{-15}$ (Figure $4 \mathrm{~B}$ ). This relation is not present for $L b$. plantarum, $\mathrm{R}^{2}=0.02, P=0.34$ (Figure $4 \mathrm{C}$ ). Differentiating based on dairy or nondairy origin (Figure $4 \mathrm{~A}$ ), or cremoris or lactis genotype (Figure 4B) does not appear to play a role in this relationship. Together this data suggests a coupling between growth and acid production in $L c$. lactis, which is not present in Lb. plantarum.

\section{DISCUSSION}

We used centrifugation to mechanically remove casein micelles from milk to facilitate direct measurements of microbial growth. We conclude that casein micelle (and other sediment) removal does have a limited effect on $L c$. lactis and Lb. plantarum growth rates or specific acidification rates. The effect is consistently found over many strains but the results indicate that the method is predictive for growth and acidification in milk. This 
A

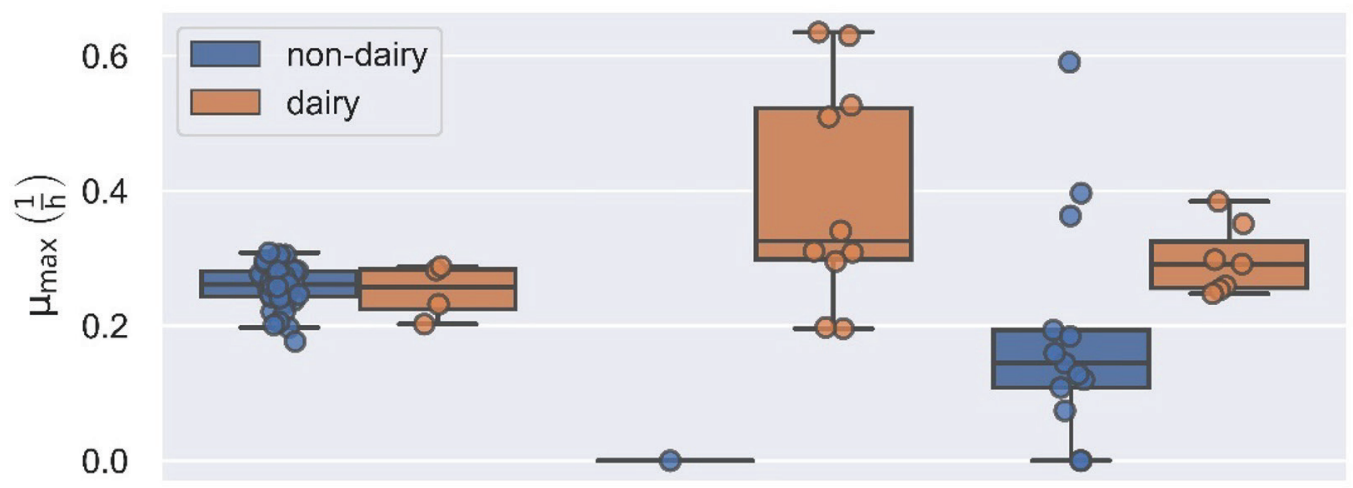

B

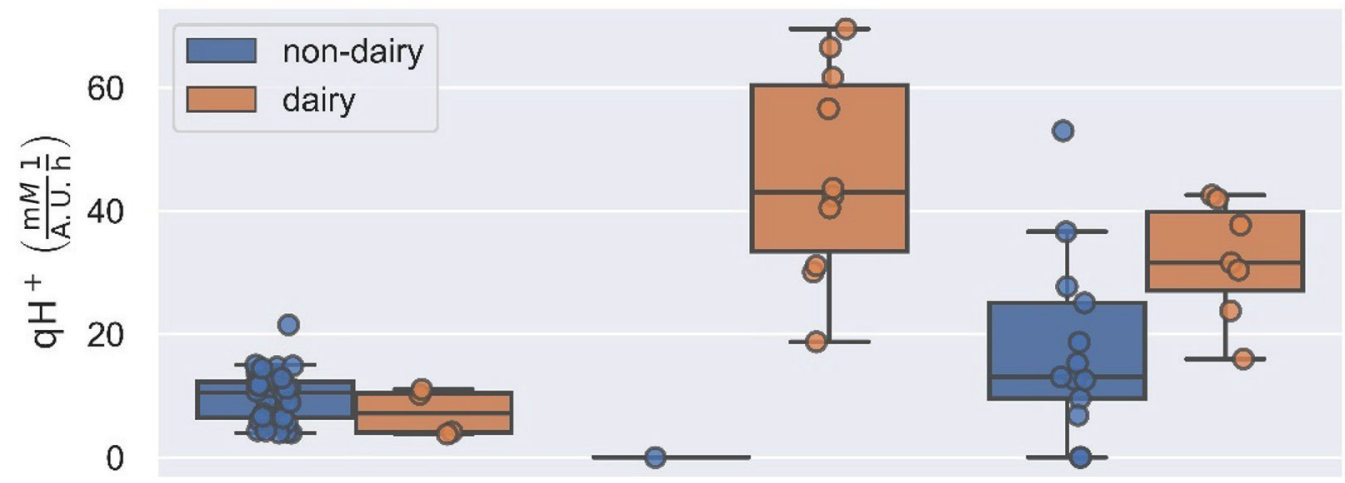

C

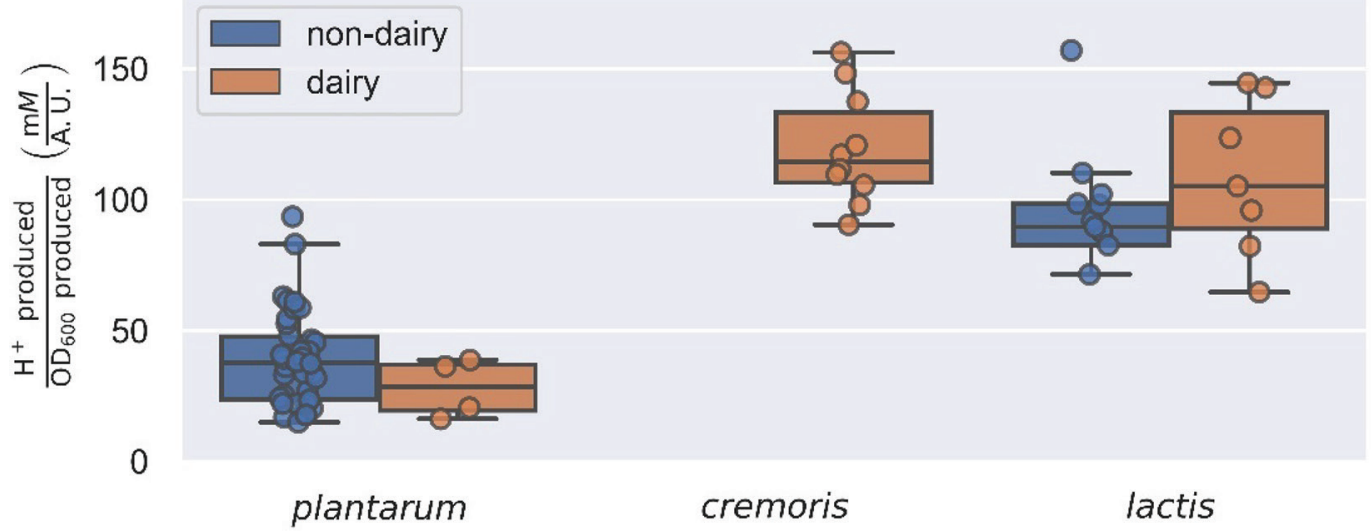

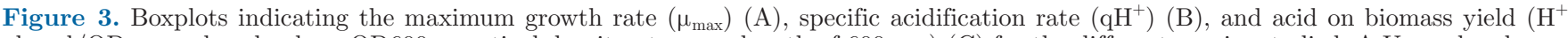
produced $/ \mathrm{OD}_{600}$ produced, where $\mathrm{OD} 600=$ optical density at a wavelength of $\left.600 \mathrm{~nm}\right)(\mathrm{C})$ for the different species studied. A.U. $=$ absorbance units. The $\mathrm{x}$-axis labels indicate the genotype, where plantarum $=$ Lactiplantibacillus plantarum, cremoris $=$ Lactococcus lactis ssp. cremoris, and lactis = Lactococcus lactis ssp. lactis. Strains were isolated from dairy (orange) or nondairy (blue) environments. Each point represents a strain average of 4 replicates (in some cases $2-3$ replicates when $\mathrm{R}^{2}<0.9$ of the linear fit to the $\log \mathrm{OD}_{600}$ data). Whiskers extend to the first and last data point not defined as an outlier. Outliers are data points that are outside the first and third quartile (box edges) by 1.5 times the interquartile range (distance between box edges).

makes it valuable for high-throughput screening (see Figure $1 \mathrm{C}$ and Figure 2).

The effect of our clearing protocol on microbial behavior cannot be fully explained by insoluble casein removal. Milk serum contains roughly $30 \%$ of the total casein at a concentration of $0.8 \% \mathrm{wt} / \mathrm{vol}$ (Rose, 1968). Additionally, Rose (1968) showed that no extra micellar casein dissolves when milk is diluted with protein- 
A All strains

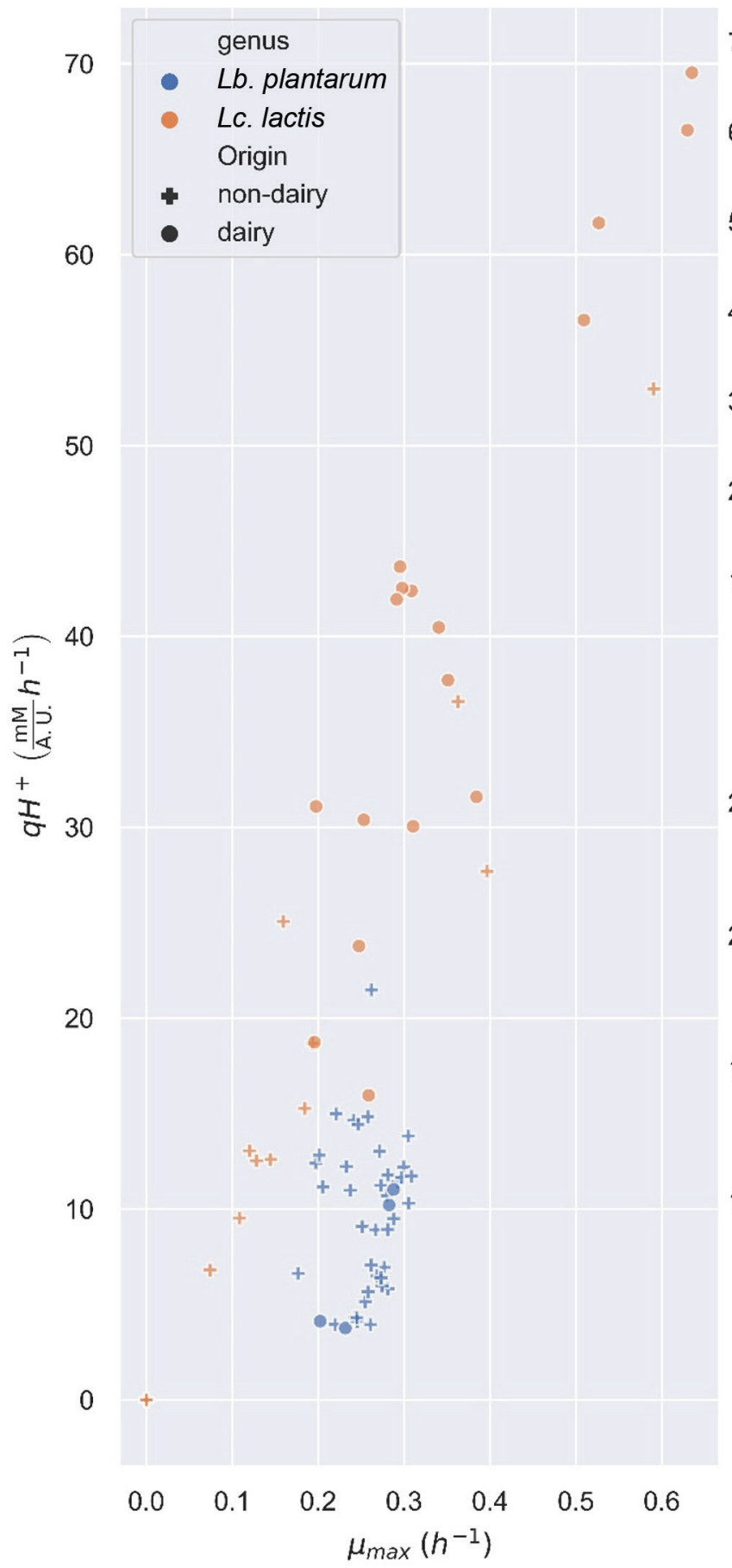

B Lc. lactis

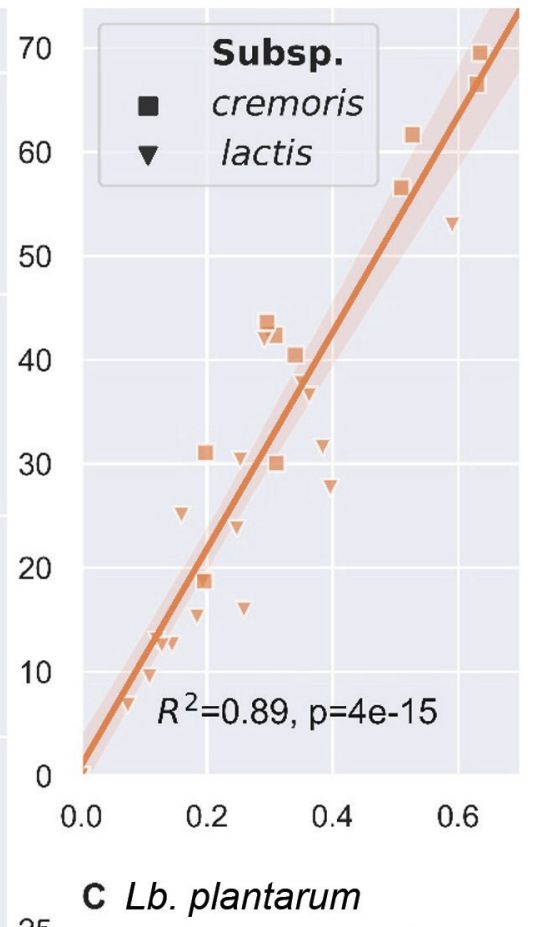

25

20

15

10

5

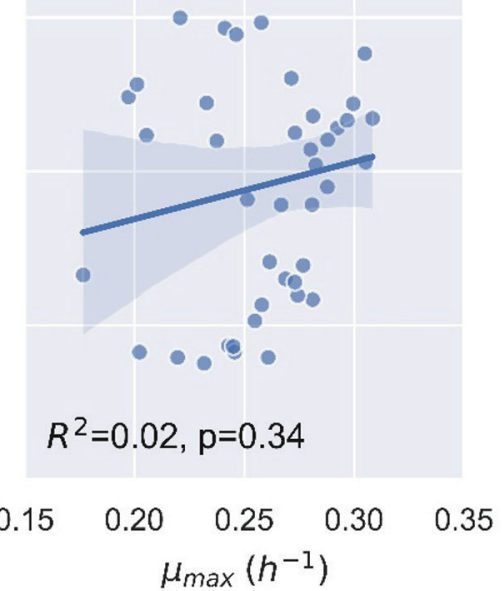

Figure 4. (A) Specific acidification rate $\left(\mathrm{qH}^{+}\right)$versus maximum growth rate $\left(\mu_{\max }\right)$ for all strains consisting of: Lactiplantibacillus plantarum (blue) and Lactococcus lactis (orange) both nondairy (plus sign) and dairy (circle) isolates. A.U. = absorbance units. (B) The same data as in panel A for Lactococcus lactis, with shape annotation indicating the subspecies, and with a linear model (solid lines), the model $\mathrm{R}^{2}$ and a 2-tailed $P$-value. (C) The same data as in panel A for Lactiplantibacillus plantarum, with a linear model (solid lines). Each point represents a strain average of 4 replicates (in some cases $2-3$ replicates when $\mathrm{R}^{2}<0.9$ of the linear fit to the $\log \mathrm{OD}_{600}$ data; $\mathrm{OD}_{600}=\mathrm{optical}_{\text {density }}$ at a wavelength of $600 \mathrm{~nm})$. 
free milk dialysate. This suggests that if the amount of casein dissolved in milk were to limit growth, the micellar undissolved casein could not replace the casein consumed from the aqueous phase. Furthermore, (Mistry and Kosikowski, 1985) showed that increasing the amount of dissolved casein does not affect the specific lactic acid production rates or the growth rates of multiple Lc. lactis and L. cremoris starter cultures (still called Streptococcus lactis and Streptococcus cremoris at the time; Schleifer et al., 1985). Higher casein concentrations do increase the buffer capacity of milk, allowing a higher growth-arresting lactic acid concentration to be reached, in addition to higher final cell density and higher final pH (Mistry and Kosikowski, 1985). This is consistent with our observations between milk serum (low buffer capacity) and milk (high buffer capacity). However, the decreased buffer capacity should only affect cell behavior near the end of the fermentation, when the $\mathrm{pH}$ drops. We cannot exclude that other relevant insoluble compounds were removed during milk clearing. One fraction that was likely reduced during milk clearing and which could affect bacterial growth is milk fat. The role of fatty acids in growth medium is demonstrated in MRS, a standard growth medium for many lactobacilli where Tween is added as a source of fatty acids. However, Tween was also shown to negatively affect growth of Lactobacillus delbrueckii in MRS (Partanen et al., 2001).

To exclude effects of unknown interactions between the carboxyfluorescein with milk solids we based our conversions from fluorescence signals to produced protons on calibration curves that were obtained in either milk or milk serum. Overall, our data shows good correlation between growth and acidification in milk serum and milk.

Literature describes several studies that assessed growth of lactic acid bacteria in milk (Siezen et al., 2010; Bayjanov et al., 2013). In Bayjanov et al. (2013) a qualitative growth score of 0,1 , or 2 was given after an unknown incubation time in milk at $30^{\circ} \mathrm{C}$. In Siezen et al. (2010), a single growth measurement was determined once after $48 \mathrm{~h}$ of incubation. Although these methods provide valuable information for genotypephenotype matching, they do not accurately assess how well different strains grow on milk. Ma et al. (2016) determined cell densities every $4 \mathrm{~h}$ by standard plate count for a subset of strains and conditions. The lack of more precise growth data in milk, such as quantitative rather than qualitative data, with more time points and not just endpoint measurements, is possibly due to a lack of appropriate measurement methods, emphasizing the value of the method presented here.

Our finding that all $L b$. plantarum strains we tested were able to grow in milk is in contrast with results reported by (Siezen et al., 2010). However, a more detailed study tested $L b$. plantarum WCFS1, ST-III, and LPHS and found growth and stationary culture pH values consistent with our study (Ma et al., 2016). Furthermore, the levels of free amino acids can vary between milk batches and increase by drying, resuspension, and sterilization of the milk (Menefee et al., 1941; Berg, 1993), which could also cause differences between various studies.

Dairy isolates are suggested to be more specialized for growth on milk than nondairy isolates (Kelly et al., 2010; Bachmann et al., 2012; Wels et al., 2019). Similarly, we see higher growth and specific acidification rates for Lc. lactis dairy isolates.

We found that the acidification rate doubles with a doubling in the growth rate for Lc. lactis. This makes sense when considering that both hetero- and homofermentative catabolic routes generate acid, and the ATP demand will likely double with a doubling of the growth rate. However, we did not find this relation for $L b$. plantarum. Considering the difference with Lc. lactis this might suggest that $L b$. plantarum strains produce only limited amounts of acidic catabolic end products in milk serum. Potentially, these strains generate part of the energy required for growth from amino acid catabolism as opposed to lactose catabolism (Konings, 2002; Teusink et al., 2006; Goffin et al., 2010). It was also suggested that $L b$. plantarum does not degrade casein but instead consumes the available free amino acids for the limited growth observed (Ma et al., 2016). This could explain us not finding a correlation between growth and acidification for this species. ATP generation through increased amino acid catabolism could be beneficial for flavor formation (Smit et al., 2009) which has been described for some Lb. plantarum strains (Di Renzo et al., 2018; Wang et al., 2020).

We conclude that milk serum is a useful alternative to milk for high-throughput strain characterization. Additionally, removing casein micelles before inoculation might benefit other bacterial characterization methods that were originally made difficult by casein interference, such as proteomics, dry weight measurements, or flow cytometry.

\section{ACKNOWLEDGMENTS}

We thank Bas Spanhaak (Vrije Universiteit Amsterdam) for laboratory assistance at the onset of this study. The project was organized by and executed under the auspices of TiFN, a public-private partnership on precompetitive research in food and nutrition. Funding for this research was obtained from Friesland Campina (Wageningen, the Netherlands), CSK Food Enrichment (Wageningen, the Netherlands) and the 
Top-sector Agri and Food. The public partners were responsible for the study design, data collection and analysis, decision to publish, and preparation of the manuscript. The private partners have contributed to the project through regular discussion. Herwig Bachman and Patrick Janssen were employed by NIZO Food Research. The remaining authors declare that the research was conducted in the absence of any commercial or financial relationships that could be construed as a potential conflict.

\section{REFERENCES}

Bachmann, H., M. Fischlechner, I. Rabbers, N. Barfa, F. Branco dos Santos, D. Molenaar, and B. Teusink. 2013. Availability of public goods shapes the evolution of competing metabolic strategies. Proc. Natl. Acad. Sci. USA 110:14302-14307. https://doi.org/10 $.1073 /$ pnas. 1308523110.

Bachmann, H., M. J. C. Starrenburg, D. Molenaar, M. Kleerebezem, and J. E. T. van Hylckama Vlieg. 2012. Microbial domestication signatures of Lactococcus lactis can be reproduced by experimental evolution. Genome Res. 22:115-124. https://doi.org/10.1101/ gr.121285.111.

Bayjanov, J. R., M. J. C. Starrenburg, M. R. Sijde, R. J. Siezen, and S. A. F. T. van Hijum. 2013. Genotype-phenotype matching analysis of 38 Lactococcus lactis strains using random forest methods. BMC Microbiol. 13:68. https://doi.org/10.1186/1471-2180-13-68.

Berg, H. E. 1993. Reactions of lactose during heat treatment of milk: A quantitative study. In Landbouw- en milieuwetenschappen. Vol. $\mathrm{PhD}$. Wageningen University, Wageningen, the Netherlands.

Brooijmans, R. J. W. 2008. Electron Transport Chains of Lactic Acid Bacteria. Page 228 in Microbiologie. PhD thesis. Microbiology Department, Wageningen University, Wageningen, the Netherlands.

Di Renzo, T., A. Reale, F. Boscaino, and M. Messia. 2018. Flavoring production in Kamut, quinoa and wheat doughs fermented by Lactobacillus paracasei, Lactobacillus plantarum, and Lactobacillus brevis: A SPME-GC/MS study. Front. Microbiol. 9:429. https:// doi.org/10.3389/fmicb.2018.00429.

Douwenga, S., P. Janssen, B. Teusink, and H. Backmann. 2021. A centrifugation-based clearing method allows high-throughput acidification and growth-rate measurements in milk. Journal of Dairy Science. Zenodo. https://doi.org/https://doi.org/10.5281/zenodo .4694160

Fowler, J. L., W. S. Clark Jr., J. F. Foster, and A. Hopkins. 1978. Analyst variation in doing the standard plate count as described in standard methods for the examination of dairy products. J. Food Prot. 41:4-7. https://doi.org/10.4315/0362-028X-41.1.4.

Fruin, J. T., T. M. Idll, J. B. Clarke, J. L. Fowler, and L. S. Guthertz. 1977. Accuracy and speed in counting agar plates. J. Food Prot. 40:596-599. https://doi.org/10.4315/0362-028X-40.9.596.

Goffin, P., B. van de Bunt, M. Giovane, J. H. J. Leveau, S. HöppenerOgawa, B. Teusink, and J. Hugenholtz. 2010. Understanding the physiology of Lactobacillus plantarum at zero growth. Mol. Syst. Biol. 6:413. https://doi.org/10.1038/msb.2010.67.

Gunasekera, T. S., P. V. Attfield, and D. A. Veal. 2000. A flow cytometry method for rapid detection and enumeration of total bacteria in milk. Appl. Environ. Microbiol. 66:1228-1232. https://doi.org/ 10.1128/AEM.66.3.1228-1232.2000.

Hunter, J. D. 2007. Matplotlib: A 2D Graphics Environment. Comput. Sci. Eng. 9:90-95. https://doi.org/10.1109/MCSE.2007.55.

Jarvis, B., A. J. Hedges, and J. E. Corry. 2007. Assessment of measurement uncertainty for quantitative methods of analysis: Comparative assessment of the precision (uncertainty) of bacterial colony counts. Int. J. Food Microbiol. 116:44-51. https://doi.org/ 10.1016/j.ijfoodmicro.2006.12.037.

John, G. T., D. Goelling, I. Klimant, H. Schneider, and E. Heinzle. 2003. pH-sensing 96-well microtitre plates for the characteriza- tion of acid production by dairy starter cultures. J. Dairy Res. 70:327-333. https://doi.org/10.1017/S0022029903006344.

Kelly, W. J., L. J. H. Ward, and S. C. Leahy. 2010. Chromosomal diversity in Lactococcus lactis and the origin of dairy starter cultures. Genome Biol. Evol. 2:729-744. https://doi.org/10.1093/ gbe/evq056.

Konings, W. N. 2002. The cell membrane and the struggle for life of lactic acid bacteria. Antonie Van Leeuwenhoek 82:3-27. https:// doi.org/10.1023/A:1020604203977.

Ma, C., G. Cheng, Z. Liu, G. Gong, and Z. Chen. 2016. Determination of the essential nutrients required for milk fermentation by Lactobacillus plantarum. LWT Food Sci Technol. 65:884-889. https:// doi.org/10.1016/j.lwt.2015.09.003.

McKinney, W. 2010. Data structures for statistical computing in Python. Pages 56-61 in Proc. 9th Python in Science Conference, Austin, TX. https://doi.org/10.25080/Majora-92bf1922-012.

Meisel, S., S. Stöckel, M. Elschner, P. Rösch, and J. Popp. 2011. Assessment of two isolation techniques for bacteria in milk towards their compatibility with Raman spectroscopy. Analyst 136:49975005. https://doi.org/10.1039/c1an15761b.

Menefee, S. G., O. R. Overman, and P. H. Tracy. 1941. The effect of processing on the nitrogen distribution in milk. J. Dairy Sci. 24:953-968. https://doi.org/10.3168/jds.S0022-0302(41)95481-4.

Mistry, V. V., and F. V. Kosikowski. 1985. Growth of lactic acid bacteria in highly concentrated ultrafiltered skim milk retentates. J. Dairy Sci. 68:2536-2543. https://doi.org/10.3168/jds.S0022 $-0302(85) 81134-6$.

Molenaar, D., F. Bringel, F. H. Schuren, W. M. de Vos, R. J. Siezen, and M. Kleerebezem. 2005. Exploring Lactobacillus Plantarum genome diversity by using microarrays. J. Bacteriol. 187:6119-6127. https://doi.org/10.1128/JB.187.17.6119-6127.2005.

Nugroho, A. D. W., M. Kleerebezem, and H. Bachmann. 2020. A novel method for long-term analysis of lactic acid and ammonium production in non-growing lactococcus lactis reveals pre-culture and strain dependence. Front. Bioeng. Biotechnol. 8:580090. https:// doi.org/10.3389/fbioe.2020.580090.

Partanen, L., N. Marttinen, and T. Alatossava. 2001. Fats and fatty acids as growth factors for Lactobacillus delbrueckii. Syst. Appl. Microbiol. 24:500-506. https://doi.org/10.1078/0723-2020-00078.

Rademaker, J. L. W., H. Herbet, M. J. C. Starrenburg, S. M. Naser, D. Gevers, W. J. Kelly, J. Hugenholtz, J. Swings, and J. E. T. van Hylckama Vlieg. 2007. Diversity analysis of dairy and nondairy Lactococcus lactis isolates, using a novel multilocus sequence analysis scheme and (GTG)5-PCR fingerprinting. Appl. Environ. Microbiol. 73:7128-7137. https://doi.org/10.1128/AEM.01017-07.

Rose, D. 1968. Relation between micellar and serum casein in bovine milk. J. Dairy Sci. 51:1897-1902. https://doi.org/10.3168/jds .S0022-0302(68)87308-4.

Savitzky, A., and M. J. E. Golay. 1964. Smoothing and differentiation of data by simplified least squares procedures. Anal. Chem. $36: 1627-1639$.

Schleifer, K. H., J. Kraus, C. Dvorak, R. Kilpper-Bälz, M. D. Collins, and W. Fischer. 1985. Transfer of Streptococcus lactis and related Streptococci to the genus Lactococcus gen. nov. Syst. Appl. Microbiol. 6:183-195. https://doi.org/10.1016/S0723-2020(85)80052-7.

Siezen, R. J., J. R. Bayjanov, G. E. Felis, M. R. van der Sijde, M. Starrenburg, D. Molenaar, M. Wels, S. A. van Hijum, and J. E. T. van Hylckama Vlieg. 2011. Genome-scale diversity and niche adaptation analysis of Lactococcus lactis by comparative genome hybridization using multi-strain arrays. Microb. Biotechnol. 4:383-402. https://doi.org/10.1111/j.1751-7915.2011.00247.x.

Siezen, R. J., V. A. Tzeneva, A. Castioni, M. Wels, H. T. K. Phan, J. L. W. Rademaker, M. J. C. Starrenburg, M. Kleerebezem, D. Molenaar, and J. E. T. van Hylckama Vlieg. 2010. Phenotypic and genomic diversity of Lactobacillus plantarum strains isolated from various environmental niches. Environ. Microbiol. 12:758-773. https://doi.org/10.1111/j.1462-2920.2009.02119.x.

Silva, M. D., K. Utvik, and M. Colantonio. 2019. Milk and milk product statistics. Pages 57-63 in Agriculture, Forestry and Fishery Statistical Book. E. Cook, ed. Eurostat. 
Smit, B. A., W. J. M. Engels, and G. Smit. 2009. Branched chain aldehydes: Production and breakdown pathways and relevance for flavour in foods. Appl. Microbiol. Biotechnol. 81:987-999. https:// doi.org/10.1007/s00253-008-1758-x.

Smith, E. M., L. E. Green, D. Mason, T. S. Gunasekera, and D. A. Veal. 2003. Savinase is a bactericidal enzyme. Appl. Environ. Microbiol. 69:719-721. https://doi.org/10.1128/AEM.69.1.719-721 2003.

Smits, H. H., A. Engering, D. van der Kleij, E. C. de Jong, K. Schipper, T. M. M. van Capel, B. A. J. Zaat, M. Yazdanbakhsh, E. A. Wierenga, Y. van Kooyk, and M. L. Kapsenberg. 2005. Selective probiotic bacteria induce IL-10-producing regulatory $\mathrm{T}$ cells in vitro by modulating dendritic cell function through dendritic cellspecific intercellular adhesion molecule 3-grabbing nonintegrin. J. Allergy Clin. Immunol. 115:1260-1267. https://doi.org/10.1016/j .jaci.2005.03.036.

Teusink, B., A. Wiersma, D. Molenaar, C. Francke, W. de Vos, R. Siezen, and E. Smid. 2006. Analysis of growth of Lactobacillus plantarum WCFS1 on a complex medium using a genome-scale metabolic model. J. Biol. Chem. 281:40041-40048. https://doi .org/10.1074/jbc.M606263200.

van der Walt, S., S. C. Colbert, and G. Varoquaux. 2011. The NumPy Array: A structure for efficient numerical computation. Comput. Sci. Eng. 13:22-30. https://doi.org/10.1109/MCSE.2011.37.

Virtanen, P., R. Gommers, T. E. Oliphant, M. Haberland, T. Reddy, D. Cournapeau, E. Burovski, P. Peterson, W. Weckesser, J. Bright, S. J. van der Walt, M. Brett, J. Wilson, K. J. Millman, N. Mayorov, A. R. J. Nelson, E. Jones, R. Kern, E. Larson, C. J. Carey, İ. Polat, Y. Feng, E. W. Moore, J. VanderPlas, D. Laxalde, J. Perktold, R. Cimrman, I. Henriksen, E. A. Quintero, C. R. Harris, A. M. Archibald, A. H. Ribeiro, F. Pedregosa, and P. van Mulbregt. 2020. SciPy 1.0: Fundamental algorithms for scientific computing in Python. Nat. Methods 17:261-272. https://doi.org/10.1038/ s41592-019-0686-2.
Wang, M., M. Lei, N. Samina, L. Chen, C. Liu, T. Yin, X. Yan, C. $\mathrm{Wu}, \mathrm{H}$. He, and C. Yi. 2020. Impact of Lactobacillus plantarum 423 fermentation on the antioxidant activity and flavor properties of rice bran and wheat bran. Food Chem. 330:127156. https://doi .org/10.1016/j.foodchem.2020.127156.

Waskom, M., O. Botvinnik, J. Ostblom, S. Lukauskas, P. Hobson, M. Gelbart, D. C. Gemperline, T. Augspurger, Y. Halchenko, J. B. Cole, J. Warmenhoven, J. de Ruiter, C. Pye, S. Hoyer, J. Vanderplas, S. Villalba, G. Kunter, E. Quintero, P. Bachant, M. Martin, K. Meyer, C. Swain, A. Miles, T. Brunner, D. O'Kane, T. Yarkoni, M. L. Williams, and C. Evans. 2020. seaborn: v0.10.1 ed. Zenodo. https://doi.org/http://doi.org/10.5281/zenodo.3767070.

Wegkamp, H. B. A. and P. C. Reemst. 2018. Ph indicator systems. The Netherlands Pat. No. WO2018197042A1.

Wels, M., R. Siezen, S. van Hijum, W. J. Kelly, and H. Bachmann. 2019. Comparative genome analysis of Lactococcus lactis indicates niche adaptation and resolves genotype/phenotype disparity Front. Microbiol. 10:4. https://doi.org/10.3389/fmicb.2019.00004.

Zheng, J., S. Wittouck, E. Salvetti, C. M. A. P. Franz, H. M. B. Harris, P. Mattarelli, P. W. O'Toole, B. Pot, P. Vandamme, J. Walter, K. Watanabe, S. Wuyts, G. E. Felis, M. G. Gänzle, and S. Lebeer. 2020. A taxonomic note on the genus Lactobacillus: Description of 23 novel genera, emended description of the genus Lactobacillus Beijerinck 1901, and union of Lactobacillaceae and Leuconostocaceae. Int. J. Syst. Evol. Microbiol. 70:2782-2858. https://doi.org/ 10.1099/ijsem.0.004107.

\section{ORCIDS}

Bas Teusink ํㅏ https://orcid.org/0000-0003-3929-0423

Herwig Bachmann ำ https://orcid.org/0000-0002-8224-0993 